\title{
A Retrospective Study of Patients with Diverticulitis: Does Neutrophil-to-Lymphocyte Ratio Predict Chronic Diverticulitis Disease Progression?
}

\author{
(1) Ali Emre Naycı, (1) Ensar Çakır \\ University of Health Sciences Turkey, İstanbul Training and Research Hospital, Clinic of General Surgery, i̇stanbul, Turkey
}

\begin{abstract}
Introduction: Diverticular disease is defined as the presence of an asymptomatic diverticulum in the colon, and an infected diverticulum is called diverticulitis. Among patients with diverticular disease, 10-25\% experience diverticulitis at some stage in their lives. This study aimed to evaluate the neutrophil-to-lymphocyte ratio (NLR) determined by dividing the neutrophil value by the lymphocyte value by comparing the stages of the patients according to the Hinchey classification who presented to the emergency department and were diagnosed with acute diverticulitis and to make a decision whether the patient should be treated by hospitalization or in the outpatient clinic.

Methods: Patients who were admitted to the Istanbul Training and Research Hospital, between 2015 and 2019 and were diagnosed with acute diverticulitis by abdominal computed tomography were included in the study. Patients aged $\leq 18$ years, pregnant patients, and patients with Crohn's disease, ulcerative colitis, colorectal, and/or anal cancer were excluded from the study.

Results: Age, white blood cell count, hemoglobin, hematocrit, platelet, monocyte, granulocyte, neutrophil, NLR, C-reactive protein, and procalcitonin values were compared according to their stage. The neutrophil and NLR values were lower in patients with stage 1 than in those with stages 3 and 4. Moreover, the neutrophil and NLR values were lower in stage 2 than in stage 3.

Conclusion: In our study, patients with acute diverticulum with higher NLR levels are more likely to develop complications. The combined use of NLR with physical examination, imaging, and other laboratory tests facilitates the diagnosis of complicated acute diverticulitis. However, further studies should be performed to confirm the utility of the NLR value in clinical practice.
\end{abstract}

Keywords: Diverticulitis, neutrophil/lymphocyte ratio, hynchey classification

\section{Introduction}

Diverticular disease, once rarely diagnosed, is now one of the most frequent gastrointestinal disorders among inpatients and outpatients (1-3). Painter and Burkitt first demonstrated a large increase in the prevalence of diverticular disease that began during the industrial revolution and documented differences in prevalence between Western and Eastern countries. In the last two decades, interest in diverticular disease has increased once again. Widespread use of modern imaging techniques like computed tomography (CT) (Figure 1) and colonoscopy and accurate diagnosis of diverticulitis and asymptomatic diverticular disease allow understanding their epidemiology (2).

Asymptomatic diverticular disease is often noticed during another imaging method. There is no consensus on the treatment and followup of the disease, as there is no clinical finding in patients without symptoms (4).
Diverticular disease is defined as the presence of an asymptomatic diverticulum in the colon, and the infection of these diverticula is called diverticulitis. Of the entire population with diverticular disease, 10-25\% experience diverticulitis at some stage in their lives (4).

An inflammation of a colonic diverticulum is also considered diverticulitis. This process could be either acute or chronic (5). In its pathophysiology, the diverticulum orifice is obstructed by a plug such as fecalith. This condition may lead to variable clinical manifestations ranging from simple diverticulitis to complicated diverticulitis, such as colonic obstruction (6). Diverticulitis can be clinically classified as complicated and non-complicated. Complicated diverticulitis usually presents with abscess formation, fistula, obstruction, or perforation (6).

The decision of whether to hospitalize the patient is an important consideration in the management of diverticulitis. The American Society for Colon and Rectal Surgery stated that several factors influence

Address for Correspondence: Ali Emre Naycı MD, University of Health Sciences Turkey, İstanbul Training and Research

Received: 27.11 .2021 Hospital, Clinic of General Surgery, İstanbul, Turkey

Phone: +90 5326487733 E-mail: aliemrenayci@gmail.com ORCID ID: orcid.org/0000-0001-8029-443X

Accepted: 19.01 .2022

Cite this article as: Naycı AE, Çakır E. A Retrospective Study of Patients with Diverticulitis: Does Neutrophil-toLymphocyte Ratio Predict Chronic Diverticulitis Disease Progression?. İstanbul Med J 2022; 23(1): 69-73.

(C) Copyright 2022 by the University of Health Sciences Turkey, Istanbul Training and Research Hospital/istanbul Medical Journal published by Galenos Publishing House. 


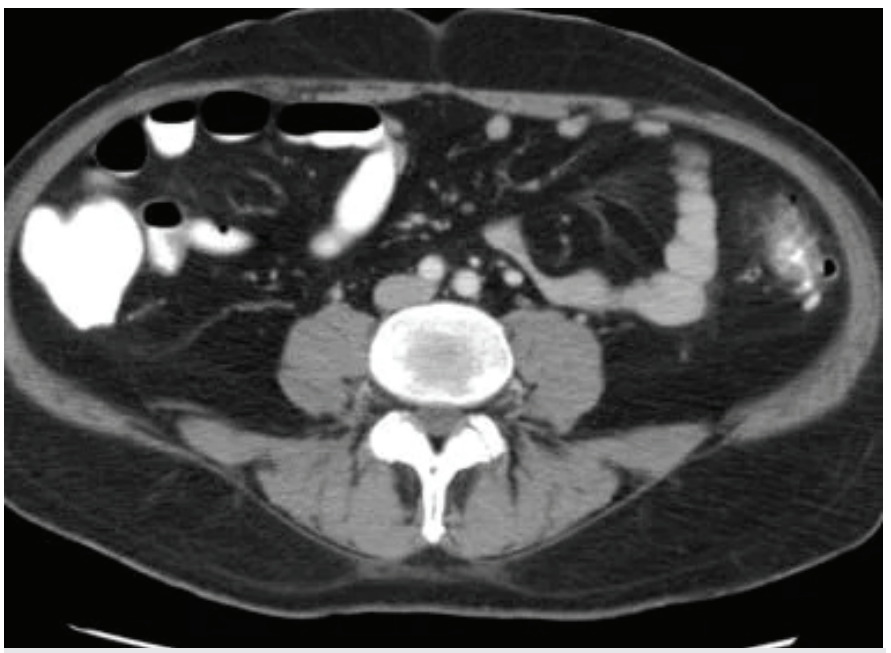

Figure 1. Diverticulitis on computed tomography

this decision, including oral intake intolerance, pain level, general comorbidities, and social support at home (7).

In recent years, its prevalence has been increasing worldwide, especially in Western countries, probably due to lifestyle changes. Although the left-sided colonic diverticular disease is more common among elderly patients, a dramatic increase has been observed in its incidence in younger age groups in recent years. Studies have shown that the lifetime risk of developing acute left-sided colonic diverticulitis in patients with diverticulosis is approximately 4\%. Data from Western societies indicate that one-fifth of the patients with acute diverticulitis are under the age of $50(8,9)$.

Patients with acute or chronic exacerbation of diverticulitis present with different clinical manifestations. The most common are left lower quadrant pain, nausea, vomiting, fever, and impaired oral intake. Leukocytosis, neutrophilia, and increased C-reactive protein (CRP) are observed in laboratory findings frequently. Although some patients with diverticulitis require hospitalization as has been stated above, the identification of patients who are likely to progress to complicated diverticulitis would help the clinician.

In this study, we aimed to evaluate the neutrophil-to-lymphocyte ratio (NLR), determined through dividing the neutrophil value by the lymphocyte value, by comparing the stages of the patients according to the Hinchey classification who presented to the emergency department and were diagnosed with acute diverticulitis and to make a decision whether the patient should be treated by hospitalization or in the outpatient clinic.

\section{Methods}

The patients who were admitted to the University of Health Sciences Turkey, İstanbul Training and Research Hospital, between 2015 and 2019 and were diagnosed with acute diverticulitis by abdominal CT were included in the study. Patients aged $<18$ years, pregnant patients, and patients with Crohn's disease, ulcerative colitis, colorectal, and/or anal cancer were excluded from the study.

The approval form the University of Health Sciences Turkey, İstanbul Training and Research Hospital Local Ethics Committee was obtained (approval number: 2421, date: 12.06.2020). Informed consent was obtained from each patient.

\section{Statistical Analysis}

Data were statistically analyzed using SPSS version 17.0. Histogram plots and the Kolmogorov-Smirnov test were employed to examine the conformity of the variables to the normal distribution. The descriptive analysis was presented using mean, standard deviation, median, and 25-75 percentile values. Categorical variables were compared by the Pearson chi-square test. The Kruskal-Wallis test was utilized to evaluate the differences between the stages in non-normally distributed (nonparametric) variables, and $p$-values $<0.05$ were regarded as significant. A total of 217 patients, including 122 men and 95 women, participated in the study.

\section{Results}

All patients were grouped according to the Hinchey classification based on tomographic imaging (Table 1). The examination of the Hinchey stages revealed that 176 patients were in stage 1, 22 in stage 2, 16 in stage 3 , and 3 in stage 4 . Moreover, 19 patients underwent surgery, 4 had drainage, 106 were hospitalized for $>5$ days, and 7 died (Table 2).

The mean age of the patients was $58.77 \pm 14.50$ years. White blood cell count (WBC), hemoglobin ( $\mathrm{Hb})$, hematocrit (HCT), platelet (PLT), monocyte, granulocyte, neutrophil, NLR, CRP, and procalcitonin values are given in Table 3.

Patients were compared according to their Hinchey stages in terms of gender, surgery, drainage, hospitalization, and mortality rates (Table 4). The surgery rate was lower in patients at stage 1 than in those at stage 3. The rate of undergoing surgery was higher in stage 4 than in other stages. The drainage rate was lower in stage 1 than in stage 3 . The rate of hospitalization for $>5$ days in stage 1 was lower than that in other

\section{Table 1. Hinchey classification \\ Hinchey classification \\ I. Pericolic abscess or phlegmon \\ II. Pelvic, intraabdominal, or retroperitoneal abscess \\ III. Generalized purulent peritonitis \\ IV. Generalized fecal peritonitis}

Table 2. Clinical data of the patients

\begin{tabular}{|l|l|l|}
\hline & $\mathbf{n}$ & $\%$ \\
\hline Male & 122 & $(56.22)$ \\
\hline Female & 95 & $(43.78)$ \\
\hline Stage 1 & 176 & $(81.11)$ \\
\hline Stage 2 & 22 & $(10.14)$ \\
\hline Stage 3 & 16 & $(7.37)$ \\
\hline Stage 4 & 3 & $(1.38)$ \\
\hline Operation & 19 & $(8.76)$ \\
\hline Drainage & 4 & $(1.84)$ \\
\hline >5-day hospitalization & 106 & $(48.85)$ \\
\hline Mortality & 7 & $(3.23)$ \\
\hline
\end{tabular}


groups. The mortality rate was significantly lower in stage 1 than in stage 4.

Age, WBC, Hb, HCT, PLT, monocyte, granulocyte, neutrophil, NLR, CRP, and procalcitonin values of the patients were compared according to their stage (Table 5). The WBC count of patients at stage 3 was higher

\section{Table 3. Complete blood count values of the patients}

\begin{tabular}{|l|l|l|l|l|}
\hline & Mean & Median & P25 & P75 \\
\hline WBC & 10.79 & 10.40 & 8.10 & 13.27 \\
\hline Hb & 13.07 & 13.30 & 11.80 & 14.40 \\
\hline HCT & 39.03 & 39.90 & 36.30 & 42.50 \\
\hline PLT & 271.85 & 263.00 & 209.00 & 313.00 \\
\hline Monocytes & 0.80 & 0.76 & 0.56 & 1.02 \\
\hline Granulocyte & 2.22 & 2.17 & 1.64 & 2.77 \\
\hline Neutrophil & 7.56 & 6.90 & 4.79 & 9.44 \\
\hline NLR & 4.33 & 3.04 & 2.12 & 4.80 \\
\hline CRP & 18.30 & 6.00 & 2.60 & 21.00 \\
\hline Procalcitonin & 1.68 & 0.50 & 0.01 & 1.60 \\
\hline
\end{tabular}

WBC: White blood cells, Hb: hemoglobin, HCT: hematocrit, PLT: platelet, NLR: neutrophil-to-lymphocyte ratio, CRP: C-reactive protein

Table 4. Gender, surgery, drainage, and mortality rates were compared according to the stage of the patients

\begin{tabular}{|c|c|c|c|c|c|c|c|c|c|}
\hline \multirow{2}{*}{$\mathrm{n}$} & & \multicolumn{2}{|c|}{ Stage 1} & \multicolumn{2}{|c|}{ Stage 2} & \multicolumn{2}{|c|}{ Stage 3} & \multicolumn{2}{|c|}{ Stage 4} \\
\hline & & $\%$ & $n$ & $\%$ & $\mathrm{n}$ & $\%$ & $\mathrm{n}$ & $\%$ & \\
\hline Gender & Male & 99 & $(56.25)$ & 12 & $(54.55)$ & 8 & $(50.00)$ & 3 & $(100.00)$ \\
\hline \multirow{2}{*}{ Operation } & No & 169 & $(96.02)$ & 19 & (86.36) & 10 & (62.50) & 0 & $(0.00)$ \\
\hline & Yes & 7 & (3.98) & 3 & (13.64) & 6 & $(37.50)$ & 3 & $(100.00)$ \\
\hline \multirow{2}{*}{ Hospitalization } & $>5$ days & 103 & $(58.52)$ & 7 & (31.82) & 1 & (6.25) & 0 & $(0.00)$ \\
\hline & $<5$ days & 73 & (41.48) & 15 & (68.18) & 15 & (93.75) & 3 & $(100.00)$ \\
\hline \multirow{2}{*}{ Mortality } & No & 173 & (98.30) & 21 & $(95.45)$ & 14 & $(87.50)$ & 2 & $(66.67)$ \\
\hline & Yes & 3 & $(1.70)$ & 1 & $(4.55)$ & 2 & $(12.50)$ & 1 & (33.33) \\
\hline
\end{tabular}

Table 5. Complete blood count values, NLR, procalcitonin, and CRP values were compared according to the stage of the patients

\begin{tabular}{|c|c|c|c|c|c|}
\hline & Stage 1 & Stage 2 & Stage 3 & Stage 4 & \\
\hline & Mean $\pm S D$ & Mean $\pm S D$ & Mean \pm SD & Mean $\pm S D$ & $\mathbf{p}$ \\
\hline & $58.13 \pm 13.66$ & $65.14 \pm 18.51$ & $57.88 \pm 15.79$ & $54.33 \pm 19.86$ & 0.392 \\
\hline WBC & $10.34 \pm 3.63$ & $10.69 \pm 3.16$ & $14.57 \pm 3.93$ & $18.23 \pm 5.51$ & 0.000 \\
\hline $\mathrm{Hb}$ & $13.25 \pm 1.89$ & $12.26 \pm 1.81$ & $12.40 \pm 1.88$ & $12.13 \pm 1.79$ & 0.043 \\
\hline $\mathrm{HCT}$ & $39.42 \pm 4.95$ & $36.92 \pm 4.63$ & $37.86 \pm 4.76$ & $37.90 \pm 2.91$ & 0.061 \\
\hline PLT & $262.93 \pm 79.88$ & $299.50 \pm 75.42$ & $312.87 \pm 149.64$ & $373.67 \pm 110.14$ & 0.006 \\
\hline Monocytes & $0.79 \pm 0.33$ & $0.89 \pm 0.37$ & $0.84 \pm 0.36$ & $0.96 \pm 0.64$ & 0.604 \\
\hline Granulocyte & $2.25 \pm 0.82$ & $2.25 \pm 0.80$ & $1.99 \pm 1.03$ & $1.30 \pm 0.70$ & 0.178 \\
\hline Neutrophil & $7.06 \pm 3.26$ & $7.35 \pm 3.03$ & $11.81 \pm 3.99$ & $15.85 \pm 5.94$ & 0.000 \\
\hline NLR & $3.84 \pm 3.91$ & $3.88 \pm 2.71$ & $8.33 \pm 7.64$ & $14.94 \pm 8.63$ & 0.000 \\
\hline CRP & $14.31 \pm 20.37$ & $19.74 \pm 21.12$ & $45.31 \pm 66.63$ & $97.67 \pm 130.20$ & 0.013 \\
\hline Procalcitonin & $1.40 \pm 2.63$ & $2.03 \pm 3.45$ & $4.04 \pm 6.07$ & $2.87 \pm 1.58$ & 0.001 \\
\hline
\end{tabular}


In general, WBC counts, procalcitonin, and CRP levels are elevated in patients with diverticulitis. In one study, the combination of high WBC count and CRP value was associated with a four-fold increased likelihood of diverticulitis compared with other causes of abdominal pain (12).

Diagnosing diverticulitis by clinical examination may be difficult for patients without a previous history of diverticulitis. Previous studies have indicated that the clinical diagnosis was incorrect in $40-60 \%$ of the patients with suspected diverticulitis. Thus, various auxiliary diagnostic tests should be implemented (13).

It may be difficult to diagnose diverticulitis based solely on clinical findings. Besides, complications of diverticulitis such as abscess cannot be confirmed without imaging examination. Differentiating the complicated diverticulitis from the uncomplicated one is very important to determine the need for antibiotics, percutaneous abscess drainage, and surgery (14).

Abdominal tomography is the most preferred imaging method in the diagnosis of diverticulitis, with a sensitivity of $96 \%$ and a specificity of $95 \%$. Abdominal ultrasonography, with sensitivity and specificity of approximately $90 \%$, can be used in the evaluation of patients with suspected diverticulitis. Ultrasonography does not require contrast, radiation is not applied, and it can be performed at the bedside. The sensitivity and specificity of magnetic resonance imaging are also high. Plain abdominal radiography may help in the evaluation of pneumoperitoneum and exclude other diagnoses such as intestinal obstruction, but it cannot be used to confirm the diagnosis of diverticulitis or abscess (15).

In a study of 177 patients with acute cholecystitis in which NLR was used as a biomarker, as in our study, NLR values were determined to be effective in the diagnosis and determination of disease severity (16). In another study of 600 patients with acute cholecystitis, NLR was successful in the diagnosis and determination of disease severity (17).

High NLR values were reported to be associated with severe abdominal infections and worse outcomes; thus, it was used as a predictor of outcomes in patients who underwent surgery (18).

Currently, the debate on the usefulness of NLR as a predictor of complications in acute diverticulitis remains open, especially concerning disease severity, clinical impact, and the need for minimally invasive or emergency surgical procedures (19).

If the CRP value in patients with diverticulitis is $>150$, it is always necessary to perform CT; if $<150$, performing $C T$ is decided according to the clinical condition of the patient (20).

The peripheral blood NLR has been widely reported to be associated with an inflammatory response and indicates the inflammatory state of many diseases. NLR is more significant as a biomarker than PLT and CRP, especially in advanced-stage acute diverticulitis.

\section{Study Limitations}

Patients aged $<18$ years, pregnant patients, and patients with Crohn's disease, ulcerative colitis, colorectal, and/or anal cancer were excluded from the study.

\section{Conclusion}

In this study, patients with acute diverticulitis with higher NLR levels are more likely to develop complications. The combined use of NLR with physical examination, imaging, and other laboratory tests facilitates the diagnosis of complicated acute diverticulitis. To confirm the utility of the NLR value in clinical practice, further studies and meta-analysis are needed.

Ethics Committee Approval: The approval form the University of Health Sciences Turkey, İstanbul Training and Research Hospital Local Ethics Committee was obtained (approval number: 2421, date: 12.06.2020). Informed consent was obtained from each patient.

Informed Consent: Informed consent was obtained from each patient.

Peer-review: Externally peer-reviewed.

Authorship Contributions: Surgical and Medical Practices - A.E.N.; Concept - A.E.N.; Design - A.E.N.; Data Collection or Processing - E.Ç.; Analysis or Interpretation - A.E.N., E.C..; Literature Search - A.E.N., E.C..; Writing - A.E.N.

Conflict of Interest: No conflict of interest was declared by the authors.

Financial Disclosure: The authors declared that this study received no financial support.

\section{References}

1. Peery AF, Crockett SD, Barritt AS, Dellon ES, Eluri S, Gangarosa LM, et al. Burden of Gastrointestinal, Liver, and Pancreatic Diseases in the United States. Gastroenterology 2015; 149: 1731-41.

2. Regenbogen SE, Hardiman KM, Hendren S, Morris AM. Surgery for diverticulitis in the 21st century: a systematic review. JAMA Surg 2014; 149: 292-303.

3. Peery AF, Dellon ES, Lund J, Crockett SD, McGowan CE, Bulsiewicz WJ, et al. Burden of gastrointestinal disease in the United States: 2012 update. Gastroenterology 2012; 143: 1179-87.

4. Hughes LE. Postmortem survey of diverticular disease of the colon. II. The muscular abnormality of the sigmoid colon. Gut 1969; 10 : 344-51.

5. Parks TG. Natural history of diverticular disease of the colon. Clin Gastroenterol 1975; 4: 53-69.

6. Bucket TP, Stollman NH. Diverticular disease of the colon. In: Feldman M, Friedman LS, Brandt LJ, editors. Sleisenger and Fordtran's gastrointestinal and liver disease: pathophysiology, diagnosis, management. 10th ed. Vol. 2. Philadelphia: Elsevier; 2014. pp. 1-15

7. Feingold D, Steele SR, Lee S, Kaiser A, Boushey R, Buie WD, et al. Practice parameters for the treatment of sigmoid diverticulitis. Dis Colon Rectum 2014; 57: 284-94.

8. Weizman AV, Nguyen GC. Diverticular disease: epidemiology and management. Can J Gastroenterol 2011; 25: 385-9. 
9. Jamal Talabani A, Lydersen S, Endreseth BH, Edna TH. Major increase in admission- and incidence rates of acute colonic diverticulitis. Int J Colorectal Dis 2014; 29: 937-45.

10. Swanson SM, Strate LL. Acute Colonic Diverticulitis Ann Intern Med 2018; 168: ITC65-80.

11. Cohen E, Fuller G, Bolus R, Modi R, Vu M, Shahedi K, et al. Increased risk for irritable bowel syndrome after acute diverticulitis. Clin Gastroenterol Hepatol 2013; 11:1614-9.

12. Andeweg CS, Knobben L, Hendriks JC, Bleichrodt RP, van Goor H. How to diagnose acute left-sided colonic diverticulitis: proposal for a clinical scoring system. Ann Surg 2011; 253: 940-6.

13. Humes DJ, Spiller RC. Review article: the pathogenesis and management of acute colonic diverticulitis. Aliment Pharmacol Ther 2014; 39: 359-70.

14. Laurell H, Hansson LE, Gunnarsson U. Acute diverticulitis-clinical presentation and differential diagnostics. Colorectal Dis 2007; 9: 496-501.

15. Andeweg CS, Wegdam JA, Groenewoud J, van der Wilt GJ, van Goor $\mathrm{H}$, Bleichrodt RP. Toward an evidence-based step-up approach in diagnosing diverticulitis. Scand J Gastroenterol 2014; 49: 775-84.
16. Beliaev AM, Angelo N, Booth M, Bergin C. Evaluation of neutrophiltolymphocyte ratio as a potential biomarker for acute cholecystitis. 2017; 209: 93-101.

17. Mahmood F, Akingboye A, Malam Y, Thakkar M, Jambulingam P. Complicated Acute Cholecystitis: The Role of C-Reactive Protein and Neutrophil-Lymphocyte Ratio as Predictive Markers of Severity. Cureus 2021; 13: e13592.

18. Miyamoto RS. Inagawa, N. Sano, S. Tadano, S. Adachi, M. Yamamoto. The neutrophil-to-lymphocyte ratio (NLR) predicts short-term and long-term outcomes in gastric cancer patients. Eur J Surg Oncol 2018; 44: 607-12.

19. Determinants of treatment and outcomes of diverticular abscesses. Mali J, Mentula P, Leppäniemi A, Sallinen V. World J Emerg Surg 2019; 14: 31 .

20. Mäkelä JT, Klintrup K, Takala H, Rautio T. The role of C-reactive protein in prediction of the severity of acute diverticulitis in an emergency unit. Scand J Gastroenterol 2015; 50: 536-41. 